\title{
Correction to: Changes in the intra- and peri-cellular sclerostin distribution in lacuno-canalicular system induced by mechanical unloading
}

\author{
Ryuta Osumi $^{1} \cdot$ Ziyi Wang $^{2,3} \cdot$ Yoshihito Ishihara ${ }^{1} \cdot$ Naoya Odagaki $^{1} \cdot$ Tadahiro limura $^{4} \cdot$ Hiroshi Kamioka $^{2}(\mathbb{D}$ \\ Published online: 6 October 2020 \\ (c) The Japanese Society Bone and Mineral Research and Springer Japan KK, part of Springer Nature 2020
}

\section{Correction to: Journal of Bone and Mineral Metabolism https://doi.org/10.1007/s00774-020-01135-9}

The original article was updated.

In the original publication of the article, the left panel of Fig. 3 was published incorrectly. The correct version of Fig. 3 is provided below.

The original article can be found online at https://doi.org/10.1007/ s00774-020-01135-9.

Hiroshi Kamioka

kamioka@md.okayama-u.ac.jp

1 Department of Orthodontics, Okayama University Hospital, Okayama, Japan

2 Department of Orthodontics, Graduate School of Medicine, Dentistry and Pharmaceutical Sciences, Okayama University, 2-5-1 Shikata-cho, Okayama, Okayama 700-8558, Japan

3 Research Fellow of Japan Society for the Promotion of Science, Tokyo, Japan

4 Department of Pharmacology, Graduate School of Dental Medicine, Hokkaido University, Hokkaido, Japan 
$\mathbf{a}$

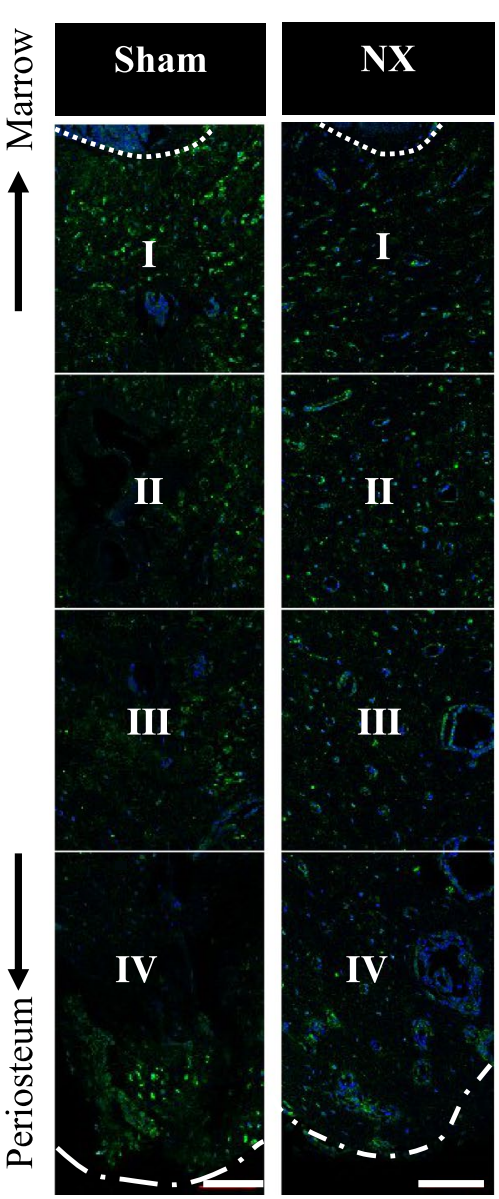

c

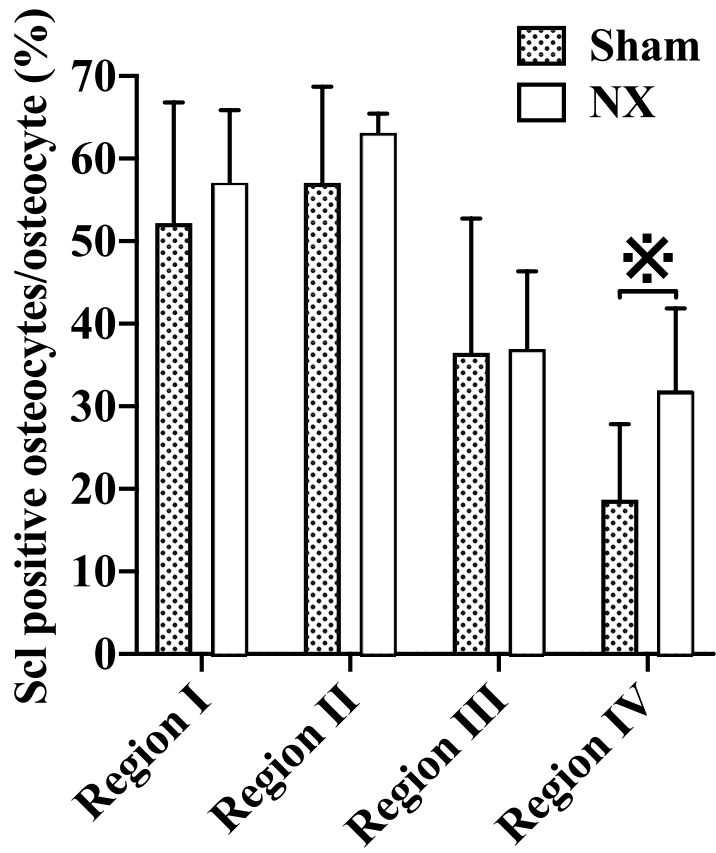

b
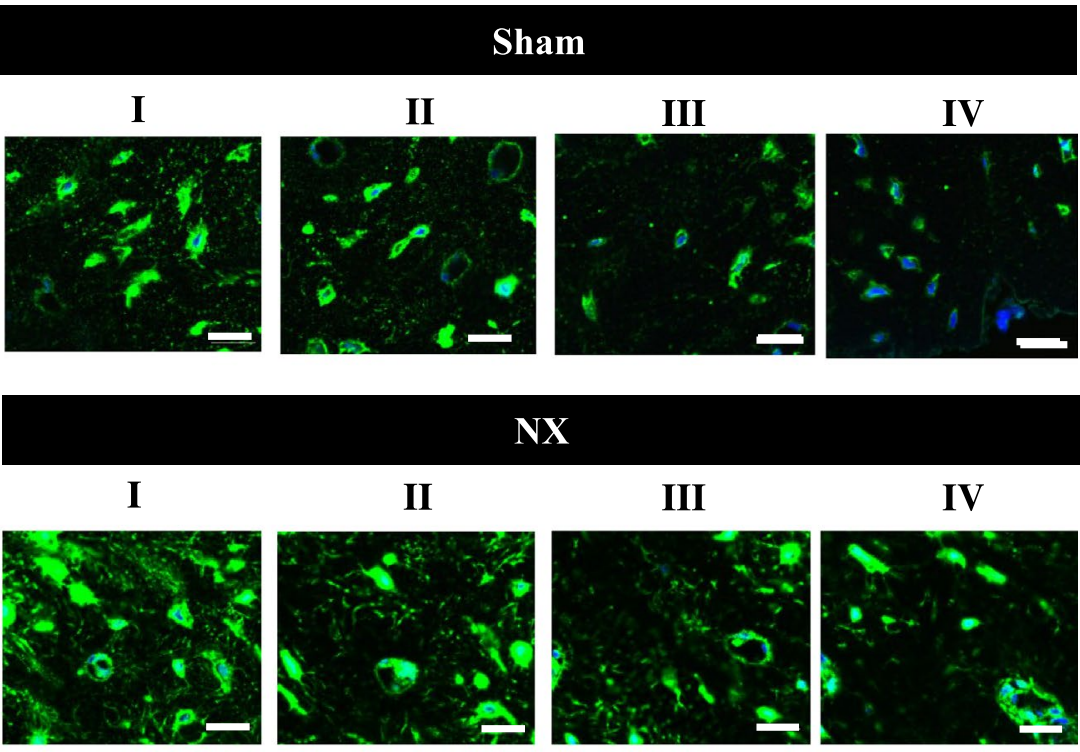

Negative Control

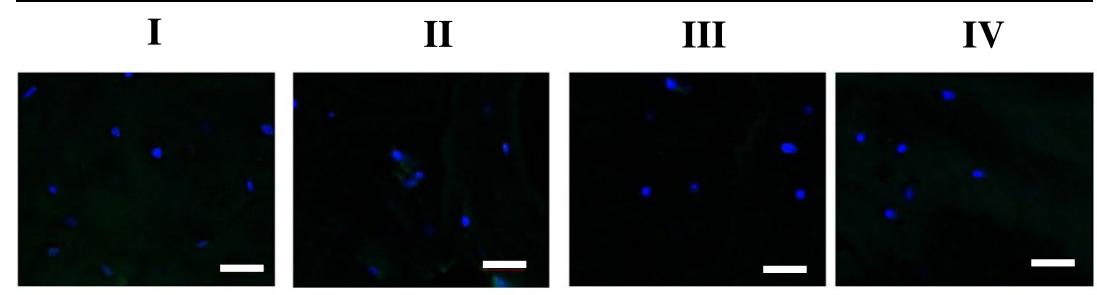

d

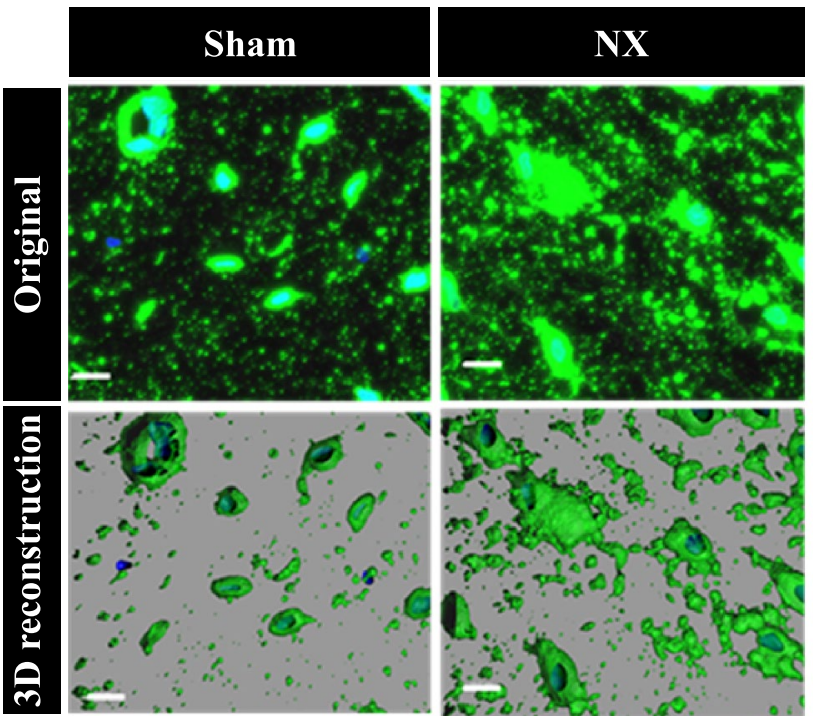


4Fig. 3 The number of sclerostin-positive osteocytes in the lateral region was evaluated. a Confocal tiling images of the lateral region. The sections were immunostained for $\mathrm{Scl}$ (green), and nuclei were stained with DAPI (blue). The white dashed line indicates the border between the marrow and cortical bone. The alternate long- and short-dash line indicates the border between the periosteal and cortical bone. The area from the marrow side to the periosteal side of the cortical bone was divided into four regions: I, II, III, and IV. Scale bar $=100 \mu \mathrm{m}$. b Magnified images of regions I, II, III, and IV. Scale bar $=20 \mu \mathrm{m}$. c The ratio of Scl-positive osteocytes to total osteocytes (nuclei stained with DAPI) in regions I, II, III, and IV. The data are expressed as the mean $\pm \mathrm{SD}(n=4)$. The asterisks indicate significant differences from the Sham group ( $※, p<0.05$ : paired $t$ test). d Threedimensional immunofluorescence images of osteocytes in regions IV constructed using the IMARIS software program. Three-dimensional fluorescence images (upper figures) were constructed using the IMARIS software program. Surface rendering images corresponding to lower figures are shown. Scl and the nuclei are indicated in green and blue, respectively. Scale bar $=10 \mu \mathrm{m}$
Publisher's Note Springer Nature remains neutral with regard to jurisdictional claims in published maps and institutional affiliations. 